\title{
Discrete Viscous Sheets
}

\author{
Christopher Batty \\ Columbia University
}

\author{
Andres Uribe \\ Columbia University
}

\author{
Basile Audoly \\ UPMC Univ. Paris 06 \& CNRS
}

Eitan Grinspun

Columbia University
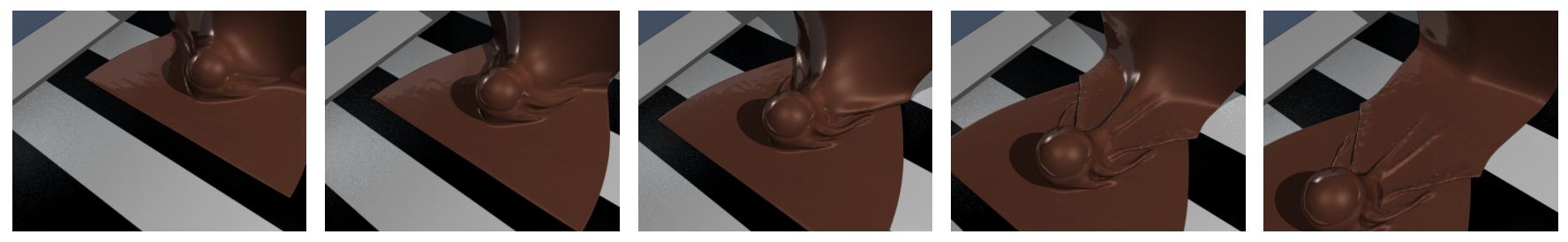

Figure 1: A thin sheet of molten chocolate enrobes a spherical truffle. As viscous sheets deform they exhibit behaviors that combine both the fluidity of liquids, and the buckling and wrinkling instabilities of thin materials, as evidenced here in the beautiful spindly legs.

\begin{abstract}
We present the first reduced-dimensional technique to simulate the dynamics of thin sheets of viscous incompressible liquid in three dimensions. Beginning from a discrete Lagrangian model for elastic thin shells, we apply the Stokes-Rayleigh analogy to derive a simple yet consistent model for viscous forces. We incorporate nonlinear surface tension forces with a formulation based on minimizing discrete surface area, and preserve the quality of triangular mesh elements through local remeshing operations. Simultaneously, we track and evolve the thickness of each triangle to exactly conserve liquid volume. This approach enables the simulation of extremely thin sheets of viscous liquids, which are difficult to animate with existing volumetric approaches. We demonstrate our method with examples of several characteristic viscous sheet behaviors, including stretching, buckling, sagging, and wrinkling.
\end{abstract}

CR Categories: I.3.7 [Computer Graphics]: Three-Dimensional Graphics and Realism-Animation;

Keywords: Viscous sheets, thin shells, fluid simulation

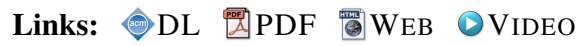

\section{Introduction}

Thin sheets of liquid give rise to an array of visually compelling phenomena, from the folding of poured cake batter to the stretching of descending chocolate sheets in a fondue fountain to the wrinkling of thin viscous films. A number of important industrial and geophysical applications also depend on the behavior of viscous sheets, including coating and enrobing processes, glass blowing and forming, lava flows, and deformations in the outer layers of the earth's crust [Ribe 2001; Ribe 2002].
Approaching the simulation of thin sheets from a volumetric vantage point remains a tremendous challenge: three-dimensional techniques commonly used for fluid animation typically break down in the limit of thin geometry. Considering the analogous case of thin elastic shells, we note that while shells can be represented with volumetric tetrahedra, in practice this is avoided, as the thin geometry necessitates excessive refinement to avoid locking and associated numerical problems [Yang et al. 2000]. The same geometric challenges arise in thin liquid sheets, motivating the development of reduced-dimensional models of thin viscous sheets.

The earliest mathematical model of a stretching two-dimensional viscous sheet was presented by Pearson and Petrie [1970]. Buckmaster, Nachman and Ting later augmented this with a bending model [Buckmaster et al. 1975], laying a foundation for multiple numerical experiments and applications [Howell 1996; Ribe 2002; Savva 2007]. All existing numerical models of viscous sheets are restricted to simple geometries, such as one-dimensional curves embedded in $\mathbb{R}^{2}$; by contrast, we seek a fully general model of two-dimensional sheets embedded in $\mathbb{R}^{3}$.

The core contribution of our work is therefore to introduce an efficient and general reduced-dimensional discrete model for viscous incompressible liquid sheets. We show that this can be done by exploiting the relationship between thin sheets and traditional shell models, allowing us to build directly on well-established techniques. We incorporate viscous effects using a simple transformation applied to elastic shell forces (the Rayleigh-Stokes analogy). We augment this model with three key features: first, we model surface tension effects with a force that seeks to minimize surface area. Second, we track the evolving thickness of sheet elements to precisely retain volume and account for the impact of thickness on fluid forces. Finally, we apply local volume-preserving remeshing operations to address the mesh quality deterioration that arises in the presence of severe stretching.

\section{Related Work}

Computational models for viscoelastic liquids and solids have become a standard tool in computer animation [Nealen et al. 2006; Bridson 2008]. We focus our discussion on the literature most relevant to viscous sheets of liquid.

Reduced dimensional models: Models based on 3D volumetric elements are typically inefficient or impractical when one or more of the physical dimensions of the material are comparatively small. By considering only the average behavior in the thin dimension(s) one can derive resultant-based methods that are more appropri- 
ate for many scenarios. This approach has been applied to twodimensional cloth and elastic thin shells [Bridson et al. 2003; Grinspun et al. 2003; Wicke et al. 2005], one-dimensional elastic rods and hair [Pai 2002; Bertails et al. 2006; Spillman and Teschner 2007; Bergou et al. 2008], and zero-dimensional elastons [Martin et al. 2010]. Similar ideas applied to thin fluid layers bounded by one or more solids give rise to shallow water equations [Kass and Miller 1990] or lubrication theory [Batchelor 1967]. Most recently, a discrete elastic rod model was extended to animate viscous threads [Bergou et al. 2010]. We pursue an analogous extension from elastic shells to viscous incompressible liquid sheets.

Volumetric methods for viscous flow: Both Lagrangian and Eulerian approaches have been applied to volumetric viscous flows. In Lagrangian mesh-based methods the mesh is evolved dynamically with the flow, and remeshing is applied in order to maintain element quality. Both viscoplastic solids [Bargteil et al. 2007; Wojtan and Turk 2008; Wicke et al. 2010] and viscous fluids [Erleben et al. 2011] have been considered under this framework, though incompressibility can give rise to locking effects. Lagrangian particle or point-based methods, such as $\mathrm{SPH}$, have also been applied to viscous and viscoplastic flows [Müller et al. 2003; Müller et al. 2004; Martin et al. 2010]. Meanwhile, Eulerian methods for viscosity have been extended to support inhomogeneous viscosity, rotation, buckling effects, and adaptivity [Carlson et al. 2002; Rasmussen et al. 2004; Batty and Bridson 2008; Batty and Houston 2011].

Thin liquid sheets: Efficiently simulating thin liquid sheets is a challenging open problem which has received a great deal of recent interest. For both Eulerian and Lagrangian mesh-based approaches, thin structures are typically dealt with either by sufficiently refining volumetric elements, at the expense of memory and computational effort (e.g., [Brochu et al. 2010]), or by embedding slender geometry within larger elements, usually at the expense of accuracy (e.g., [Wojtan et al. 2010]). A third proposed approach is to detect thin regions, and artificially inflate them before they fall below the mesh resolution and disappear [Chentanez et al. 2007]. For particle-based approaches, adaptive particle-splitting within the plane of the sheet has been used to good effect [Ando and Tsuruno 2011].

\section{Viscous Sheets}

The modeling of viscous sheets requires consideration of viscous membrane response, viscous bending response, volume conservation, and surface tension.

\subsection{Smooth setting}

In the smooth setting, the balance of linear momentum for a thin viscous sheet can be written symbolically as (see [Ribe 2002])

$$
\begin{aligned}
N_{\alpha \beta, \beta}+F_{\alpha} & =\rho h \ddot{x}_{\alpha} \\
M_{\alpha \beta, \alpha \beta}+\kappa_{\alpha \beta} N_{\alpha \beta}+F_{3} & =\rho h \ddot{x}_{3},
\end{aligned}
$$

where $(\rho h)$ is the mass density of the sheet per unit area, $\kappa_{\alpha \beta}$ the curvature tensor, $\left(x_{1}, x_{2}\right)$ is a system of coordinates along the sheet in current configuration, $x_{3}$ is a coordinate in $3 \mathrm{~d}$ space that is locally

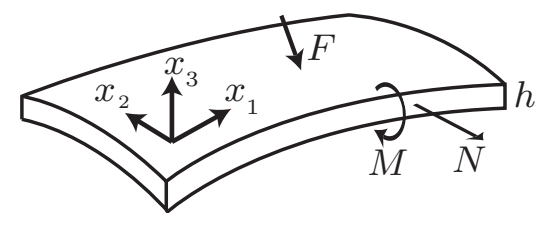

Figure 2: The geometry of a viscous sheet, as described in $\S 3.1$.

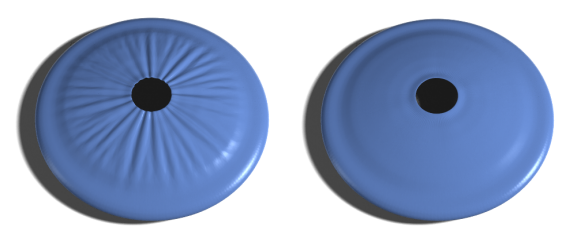

Figure 3: When a cap-shaped viscous sheet enclosing a bubble of air is punctured, it sags and forms the characteristic wrinkling pattern associated to any attempt to flatten a curved surface (left). Increasing surface tension suppresses wrinkling (right).

normal to the surface, a dot $\dot{x}=\partial x / \partial t$ denotes differentiation in time, and a subscripted comma denotes partial differentiation. By convention, Greek indices run over in-plane directions, $\alpha, \beta \in$ $\{1,2\}$. Equation (1) expresses the balance of linear momentum in the direction tangent to the surface, and (2) in the normal direction. The internal stress in the sheet are captured by two tensors, the membrane stress tensor $\mathbf{N}$ and the bending moment tensor $\mathbf{M}$. The density of applied force $\mathbf{F}$ (including surface tension and gravity effects) is combined with the resultant of internal stress in the lefthand sides, and drives the rate of change of linear momentum $\rho h \ddot{\mathbf{x}}$ in the right-hand sides. These equations of motion are valid for any two-dimensional medium, including an elastic plate.

The constitutive laws for the viscous sheet are [Ribe 2002]

$$
\begin{aligned}
N_{\alpha \beta} & =2 \mu h\left(\dot{\epsilon}_{\alpha \beta}+\delta_{\alpha \beta} \operatorname{Tr} \dot{\boldsymbol{\epsilon}}\right), \\
M_{\alpha \beta} & =-\frac{\mu h^{3}}{6}\left(\dot{\kappa}_{\alpha \beta}+\delta_{\alpha \beta} \operatorname{Tr} \dot{\boldsymbol{\kappa}}\right) .
\end{aligned}
$$

Here, $\mu$ is the dynamic viscosity of the fluid and $\dot{\boldsymbol{\epsilon}}$ and $\dot{\boldsymbol{\kappa}}$ are the strain rate tensors, measuring the rate of change of in-plane lengths, and the rate of change of curvature, respectively, in a frame moving along with the sheet. Incompressibility is taken into account by an equation of evolution for thickness $h$,

$$
\frac{\mathrm{D} h}{\mathrm{D} t}=-h \operatorname{Tr} \dot{\boldsymbol{\epsilon}}
$$

where $\mathrm{D} / \mathrm{D} t$ denotes convected derivative.

The set of equations (1-5) forms a well-posed mathematical problem governing the dynamics of thin viscous sheets. These equations are coupled, non-linear partial differential equations of degree 4 with respect to space, and 2 with respect to time; they have no analytical solutions, except in a few very simple geometries.

\subsection{Elastic shells to viscous sheets}

Triangle mesh discretization In our spatial discretization, the material domain and deformed configuration are both represented by the same triangle mesh. Each triangle carries an associated thickness, so that it represents a (time-invariant) volume of fluid. Imposing a constant volume of fluid in each triangle is a simple way of implementing the smooth equation (5), as the latter implies that the volume $(A h)$ is advected passively, $\mathrm{D}(A h) / \mathrm{D} t=0$.

Discrete Stokes-Rayleigh analogy We discretize (3) and (4) by employing the analogy proposed by Stokes [1845] and Rayleigh [1945], who observed that a viscous formulation derives from an elastic formulation when velocities replace displacements and strain rates replace strains. This observation facilitates the transformation of elastic to viscous numerical models [Baraff and Witkin 1998; Radovitzky and Ortiz 1999; Kharevych et al. 2006; Bergou et al. 2010]. 


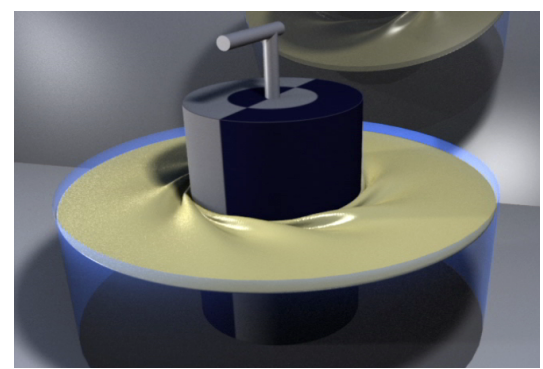

Figure 4: An annular viscous sheet floating on a bath of denser fluid develops wrinkles when sheared by rotating the inner cylinder.

Assume that an elastic potential $E(\epsilon, \bar{\epsilon})$ is expressed in terms of some strains $\epsilon$ and $\bar{\epsilon}$, measured in the deformed and undeformed configurations, respectively. Elastic forces follow from taking variations of the elastic potential with respect to the material coordinates. To obtain viscous forces in a numerical code one first formulates a discrete dissipative potential

$$
E_{\text {visc }}\left(\dot{\epsilon}_{k+1}\right)=(\nu / \Delta t) E\left(\epsilon_{k+1}, \epsilon_{k}\right),
$$

where the scalar $\nu$ governs the relative viscosity and the time interval $\Delta t$ between consecutive integration steps $\{k, k+1\}$ appears as a divisor as the result of the strain rate discretization $\dot{\epsilon}_{k+1}=\left(\epsilon_{k+1}-\epsilon_{k}\right) / \Delta t$. Viscous forces then follow from taking variations of the discrete dissipative potential with respect to the end-of-step configuration. As in the above earlier works, this formulation of viscosity requires only a trivial modification to an elastic shell implementation: a viscous update at each time step sets the undeformed to the current strain, $\bar{\epsilon}_{i}=\epsilon_{i}$, allowing code for elastic forces and Jacobians to be directly reused.

Discrete Elastic Shells Recent research on mesh-based elastic shells has improved simulation capabilities along a number of axes, including enhanced bending, inextensibility, strain-limiting, and fracture [Gingold et al. 2004; Garg et al. 2007; English and Bridson 2008; Wang et al. 2010]. For completeness, we review the potential energies for our chosen shell model, and refer the reader to references for further details. After application of the discrete Stokes-Rayleigh analogy, this model yields discrete analogues of the constitutive relations (3) and (4).

Membrane model. We use a standard linear finite element (constant strain triangle) model of in-plane stretching. Following Gingold et al. [2004], the Green strain $\varepsilon$ for a single triangle is

$$
\varepsilon=\frac{1}{16 A^{2}} \sum_{i=1}^{3}\left(l_{i}^{2}-\vec{l}_{i}^{2}\right)\left(\mathbf{t}_{j} \otimes \mathbf{t}_{k}+\mathbf{t}_{k} \otimes \mathbf{t}_{j}\right)
$$

where $A$ is triangle area, $l_{i}$ and $\bar{l}_{i}$ are the deformed and reference lengths of edge $i$, respectively, $\otimes$ indicates the outer product of two vectors, and $\mathbf{t}_{i}$ is an in-plane outward normal to edge $i$ in the undeformed state; henceforth, barred quantities distinguish undeformed (or "reference") quantities from their deformed counterparts. Assuming an isotropic linear elastic constitutive model, the potential energy summed over all triangles $t$ is

$$
E_{s}=\frac{1}{2} \sum_{t} A_{t} \frac{Y h_{t}}{\left(1-\nu^{2}\right)}\left((1-\nu) \operatorname{Tr}\left(\left(\varepsilon_{t}\right)^{2}\right)+\nu\left(\operatorname{Tr} \varepsilon_{t}\right)^{2}\right)
$$

where $Y$ is Young's modulus, $\nu$ is Poisson's ratio, and $h$ is triangle thickness. The subscript $t$ indicates the value associated to a particular triangle. We select physical parameters (§3.3) so as to establish equivalence with the viscous constitutive law (3). By variation, this quadratic stretching energy is associated with a linear, elastic constitutive law which reads $N_{\alpha \beta}=\frac{Y}{1+\nu}\left(\epsilon_{\alpha \beta}+\frac{\nu}{1-\nu} \delta_{\alpha \beta} \operatorname{Tr} \varepsilon\right)$.

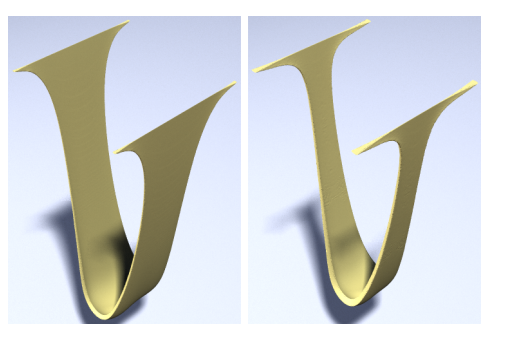

Figure 5: Two viscous catenary sheets sag under gravity. Left: A Poisson effect (horizontal contraction) is visible without surface tension. Right: Surface tension exaggerates the contraction and increases thickness.

Bending model. For bending, we use the common hinge-based model [Bridson et al. 2003; Grinspun et al. 2003]. Flexural rigidity (stiffness) of an elastic shell is given as

$$
\frac{Y h^{3}}{12\left(1-\nu^{2}\right)} \text {. }
$$

The associated discrete energy summed over all edges $e$ is

$$
E_{b}=\sum_{e} \frac{Y h_{e}^{3}}{12\left(1-\nu^{2}\right)}\left(\theta_{e}-\bar{\theta}_{e}\right)^{2} \bar{l}_{e} / \bar{l}_{e}^{*} .
$$

where $\theta_{e}$ refers to the angle between the two incident triangles, $h_{e}$ is the mean thickness of the two incident triangles, $A$ is the total area of the incident triangles, and $\bar{l}_{e}^{*}=A /\left(3 \bar{l}_{e}\right)$ is the length of the barycentric dual edge. By computing the first variation of this bending energy $E_{b}$ in the smooth limit, one finds a linear constitutive law for bending that is the elastic analogue of equation (4) with the strain rate $\dot{\kappa}_{\alpha \beta}$ replaced by the bending strain $\kappa_{\alpha \beta}$.

\subsection{Fluid Material Parameters}

To adapt the elastic shell model to the fluid sheet setting, we assign values capturing the fluid's properties to the parameters $Y$ and $\nu$. Incompressibility of the fluid gives $\nu=\frac{1}{2}$. To determine the appropriate value for $Y$, we note that the elastic analog of the fluid dynamic viscosity $\mu$ is the shear modulus, $G$, equal to $G=Y / 3$ in the incompressible case. Therefore we set $Y=3 \mu$.

\subsection{Surface Tension}

Surface tension acts to minimize surface area of a liquid [Batchelor 1967]. We model this by incorporating an additional potential energy based on the surface area of the triangles, assuming piecewise constant thickness. The resulting potential energy term is

$$
E_{s t}=\sum_{t} 2 \gamma A_{t}
$$

where $\gamma$ is the surface tension coefficient, and the factor of two accounts for the top and bottom surfaces; corresponding forces and Jacobians are derived in the supplemental material. We emphasize that under the thin sheet assumption of small thickness, the energy contributed by the exposed cross-sectional area at boundary edges of the sheet is negligible, and not responsible for the dominant retraction effects in these regions. We therefore base our discrete surface energy on the sheet's mid-surface area alone. Our approach is similar to recent area-based surface tension models for volumetric Lagrangian fluids [Misztal et al. 2010; Erleben et al. 2011], except that rather than using a linear approximation, we solve the fully nonlinear problem with Newton's method.

\subsection{Contact and Adhesion}

We model contact and adhesion by assuming zero relative velocity between colliding sheets of fluid, i.e., a no-slip condition. For solidfluid contact, we apply hard position constraints to fluid vertices 
that collide with the (possibly moving) solids. For self-collisions, we formulate a simple penalty model. A vertex and triangle are considered to be colliding when the normal component of their separation vector is less than the average of their local thicknesses. We then add a spring force consisting of two independent components: a normal spring with rest-length equal to the current normal distance, and a zero rest-length tangential spring that opposes sliding. This amounts to an anchor spring model with infinite friction [Hasegawa and Fujii 2003]. Along with penalty forces, we exploit the El Topo library's robust collision-processing module to avoid missing collisions at large time steps [Brochu and Bridson 2009].

\subsection{Triangle Thickness and Quality}

Thickness update The sheet thickness, $h$, is treated as piecewise constant over each triangle, and a triangle's volume is assumed to be $V=A h$. After each time integration step, the new area of the triangle is used to enforce incompressibility by directly updating the thickness of the triangle such that its volume is unchanged. This is a simple explicit splitting approach to the fully coupled problem of simultaneous evolution of position and thickness, under the assumption of slowly varying thickness.

Mesh adaptation Surface remeshing in elastic shell simulation is typically intended to support adaptivity, for which regular hierarchical and reversible refinement is often sufficient [Hutchinson et al. 1996; Zhang and Yuen 2001; Villard and Borouchaki 2005]. In contrast, our focus is on maintaining good mesh quality in the presence of large and irreversible stretching flows, which necessitates more general remeshing strategies even for uniform resolution. Much like triangle mesh-based surface tracking methods for volumetric fluids, we apply local remeshing operations to improve triangle quality [Wojtan et al. 2009; Brochu and Bridson 2009; Brochu et al. 2010; Zhang et al. 2011]. We apply three standard operations: edge split, edge flip, and edge collapse. The schedule of operations was dictated by the El Topo library's surface improvement module [Brochu and Bridson 2009], which we extended slightly to support operations on boundary triangles.

We exactly preserve fluid volume during each operation by manipulating triangle thicknesses. During an edge split, any new subtriangles are assigned the thickness of their parent triangle. During an edge flip, the total volume of the two triangles is computed, and re-distributed to the new triangles such that they possess the same thickness. During an edge collapse, we first sum the "lost volume" from two sources: the total volume of the triangles being deleted, and the reduction in volume of any incident triangles whose area decreases as a result of the collapse. This lost volume is then redistributed to the remaining incident triangles in proportion to the increase in their area. The remaining physical quantities (position, velocity, etc.) are treated with barycentric interpolation over triangles. While this entails numerical dissipation, our focus on the viscous regime renders such effects negligible.

\subsection{Time Integration Algorithm}

The stored energy of an elastic sheet in Lagrangian coordinates is typically defined by measuring strain relative to a two-dimensional undeformed domain. Using the Stokes-Rayleigh analogy a discrete viscous potential may be represented similarly, however, in the purely viscous setting a material lacks "history" and therefore does not carry a persistent undeformed configuration. That observation, accompanied by the notably unbounded strains that develop in a Lagrangian-tracked viscous sheet, suggests that an updated Lagrangian representation is advantageous:
At the start of each time step, the reference configuration is initialized to match the start-ofstep configuration. The discrete dissipative potentials $(\S 3.2)$ are defined with respect to this reference configuration. To avoid the stringent time step restrictions associated with viscosity and surface tension, we apply first order implicit Euler ("backward Euler") integration and solve with Newton's method. El Topo collision-processing is then applied to find a safe endof-step configuration. We next adjust each triangle's thickness to conserve volume, and then check for geometry in close proximity, adding new contact springs/constraints as needed. Finally, we apply remeshing to improve triangle quality.

\section{Results}

Stretching Validation. We validate our viscous stretching model with a spherical sheet of radius $r$ inflated by a pressure force. We ran four numerical experiments using different sheet properties and internal pressure functions $p(t)$. The simulation results are compared to the exact instantaneous velocity of expansion, $\dot{r}(t)=\frac{p(t) r^{2}(t)}{12 \mu h}$, derived in the supplemental material. The agreement is confirmed in Fig. 6 by the collapse of all numerical data onto the dashed line corresponding to the analytical prediction.

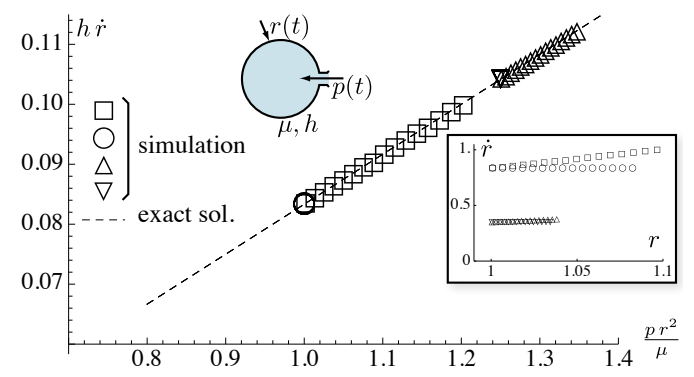

Figure 6: Validation of stretching, based on the inflation of a viscous, spherical balloon under internal pressure $p(t)$. Four simulations were run, indicated by different shapes $(\triangle \nabla \square \bigcirc)$. These correspond to a sheet of thickness $h=.1$ and viscosity $\mu=.1(\square$ $\bigcirc)$ or to $h=.3$ and $\mu=.1(\triangle \nabla)$, and to a time-independent pressure $(\square \triangle)$ or to a decreasing pressure $p(t)$ proportional to $1 / r^{2}(\bigcirc \nabla)$, where $r$ is the current sphere radius. In inset, raw data are shown in the plane $(r, \dot{r})$. In the main plot, the same data is shown in terms of rescaled coordinates $x=p r^{2} / \mu$ and $y=h \dot{r}$, and compared to the exact solution $y=x / 12$.

Surface Tension Validation. We consider the radial contraction of a unit radius, zero viscosity spherical sheet due to surface tension. Fig. 7 illustrates that for various parameter settings the time evolution of the radius is consistent with the analytical prediction $r(t)=r_{0} \cos (t / T)$ for $T=\frac{r_{0}}{2} \sqrt{\rho h_{0} / \gamma}$, derived in the supplemental material.

Sheared Wrinkling Sheet. As with cloth or shells, viscous sheets can exhibit wrinkling due to bending forces. A classic example is a sheared annular sheet of viscous liquid floating on a bath of a much heavier inviscid liquid [Benjamin and Mullin 1988], as shown in Fig. 4. The effect of the bath is approximated by a pressure force $p=\rho g d$, where $\rho$ is the density of the bath, and $d$ is the vertical displacement from the rest height. We begin by rotating 


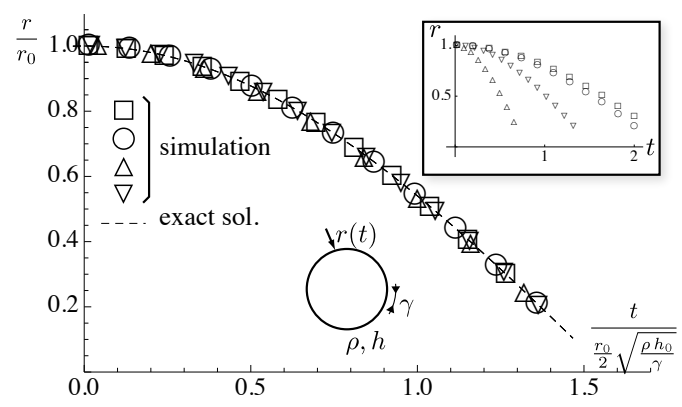

Figure 7: Validation of surface tension, based on the surface tension-driven contraction of a unit-radius inviscid spherical sheet. Four simulations were run, varying the parameter set $\left(h_{0}, \rho, \gamma\right): \triangle(0.01,1,0.001), \nabla(0.02,1.3,0.003), \square(0.05,0.2,0.01)$, and $\bigcirc(0.06,0.25,0.004)$. In inset, raw data are shown in the plane $(t, r)$. In the main plot, the same data is shown in terms of rescaled coordinates $x=t /\left(\frac{r_{0}}{2} \sqrt{\frac{\rho h_{0}}{\gamma}}\right)$ and $y=\frac{r}{r_{0}}$, and compared to the exact solution $y=\cos (x)$.

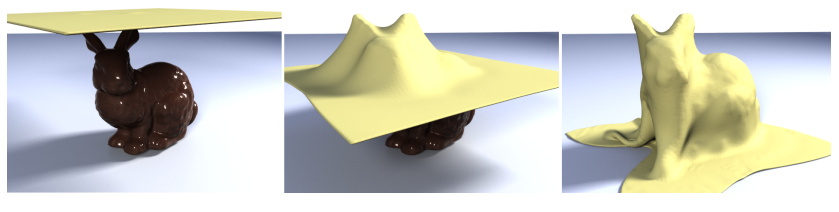

Figure 8: A rectangular viscous sheet collapses and sags over the Stanford bunny.

the inner boundary of the sheet at constant velocity until steady state wrinkling is achieved. We then steadily decrease the rotation rate, and as it crosses a critical threshold the combined smoothing effect of surface tension and the fluid bath cause the wrinkling to subside, illustrating the nonlinear character of this effect [Benjamin and Mullin 1988; Teichmann 2002]. If the rotation rate is set too low, no wrinkling develops and the sheet remains flat.

Stretching and Sagging. The supplemental video illustrates stretching and remeshing effects, by pouring a sheet of fluid vertically with constant inflow velocity and thickness. As gravity accelerates and stretches the falling sheet, incompressibility induces a Poisson effect (horizontal contraction), which is further exaggerated by surface tension. Similar effects arise for a "catenary sheet:" Fig. 5 shows a rectangular sheet held fixed along two edges and allowed to sag under gravity [Teichman and Mahadevan 2003].

Collapsing Viscous Bubble. When a high viscosity bubble resting on a liquid surface is popped, internal air pressure ceases to support it. As it collapses under gravity, periodic wrinkles form along the circumference, as shown in Fig. 3. Da Silveira et al. [2000] present a scaling argument that the number of wrinkles is determined by competition between bending and gravity, which prefer lower and higher frequency modes, respectively.

Periodic Buckling. Another characteristic behavior of viscous sheets is periodic buckling in which the sheet folds back and forth over itself [Skorobogatiy and Mahadevan 2000; Ribe 2003]. This can often be observed when pouring cake batter, for example. Similar to the fluid mechanical sewing machine of Bergou et al. [2010], we pour fluid onto a moving belt and observe pronounced folding (Fig. 10). Taking artistic license with the rheological properties of ice cream, we use a similar scenario with a slanted inflow to approximate the production of the layered folds of Viennetta ${ }^{\mathrm{TM}}$ brand ice cream (Fig. 9).
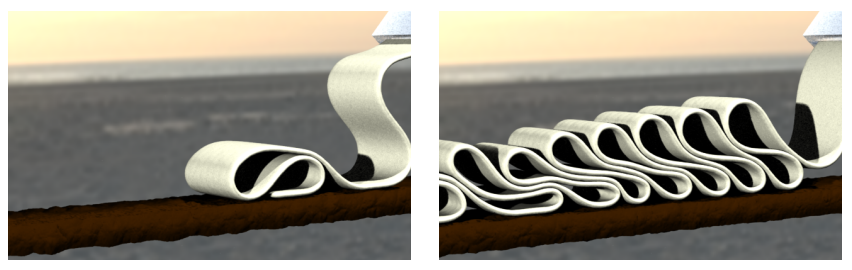

Figure 9: Uncovering the mystery of the Viennetta ${ }^{T M}$ folded ice cream production line.

\begin{tabular}{lcc}
\hline Figure & Number of vertices & Mean time per frame (s) \\
\hline 1 - Enrobing & $4010-14931$ & 18.05 \\
8 - Bunny & $3721-5328$ & 0.884 \\
9 - Viennetta & $99-3951$ & 12.77 \\
11 - Tearing & $5210-21258$ & 12.02 \\
\hline
\end{tabular}

Table 1: Performance results for some representative simulations, captured on a $1.73 \mathrm{GHz}$ laptop with a single-threaded implementation of our method.

Liquid Sheet Draping. A traditional example in cloth animation is draping over a sphere. Our supplementary video demonstrates a "viscous drape" by releasing a rectangular sheet over a sphere. Similar to cloth, large folds develop; by contrast, irreversible stretching and remeshing occurs in the area immediately surrounding the sphere. Next we illustrate support for arbitrary collision geometry by performing a similar draping test on the Stanford bunny, modeled as a signed distance field (Fig. 8). To end on a sweet note, we enrobe a truffle in molten chocolate (Fig. 1).

Tearing. We can also leverage other methods and concepts from cloth animation, such as tearing. Fig. 11 demonstrates a sphere tearing through a pouring viscous chocolate sheet at high speed, using a simple model in which we separate any edge whose incident triangles have thicknesses below a chosen threshold.

\section{Discussion}

We have presented the first general reduced dimensional numerical model for three-dimensional viscous thin sheets. It exhibits the rich behavior of such fluids, including stretching, buckling, folding, and wrinkling. We have provided validation of stretching and surface tension forces, deferring bending to future work. Our approach reduces the scalability challenges inherent in volumetric approaches and avoids locking induced by incompressibility conditions, while presenting several exciting avenues for future work.

It would be interesting to consider seamless transitions to and from lower-dimensional models (threads and droplets) and higherdimensional ones (volumes). A complete treatment could also account for non-manifold junctions between dimensionally reduced models, and new topological operations to merge colliding and stacking shells/sheets. Because we did not consider such transitions, we consider our current model of merging and adhesion to be suitable primarily for simple visual effects. Designing accurate models for the instability and breakup of liquid sheets is a related open problem.

On the other hand, the dimensionality reduction is likely to make some extensions particularly easy. For example, one could combine our approach with elastoplasticity and advanced failure (tearing/fracture/cutting) models, which are readily available for triangle meshes (e.g. [Gingold et al. 2004]). Because of the extreme distortions of the triangle mesh, this simulation domain will likely serve as an attractive test bed for novel remeshing and mesh-adaptation 

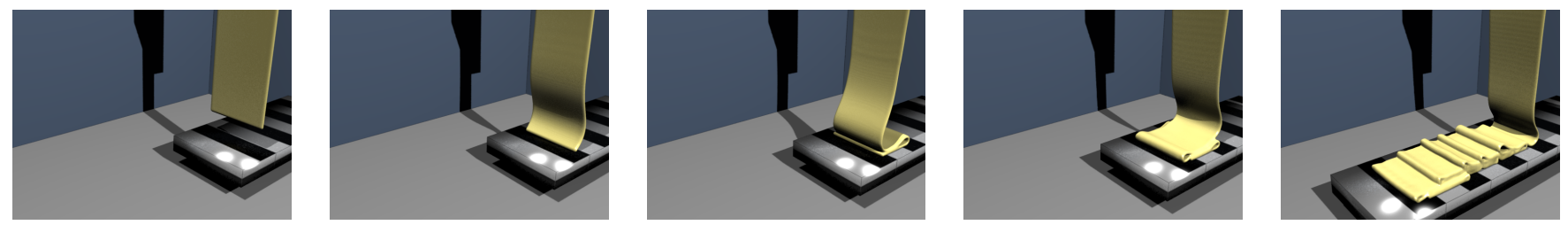

Figure 10: A sheet of viscous liquid extruded onto a moving conveyor belt first buckles, and then begins to fold periodically.
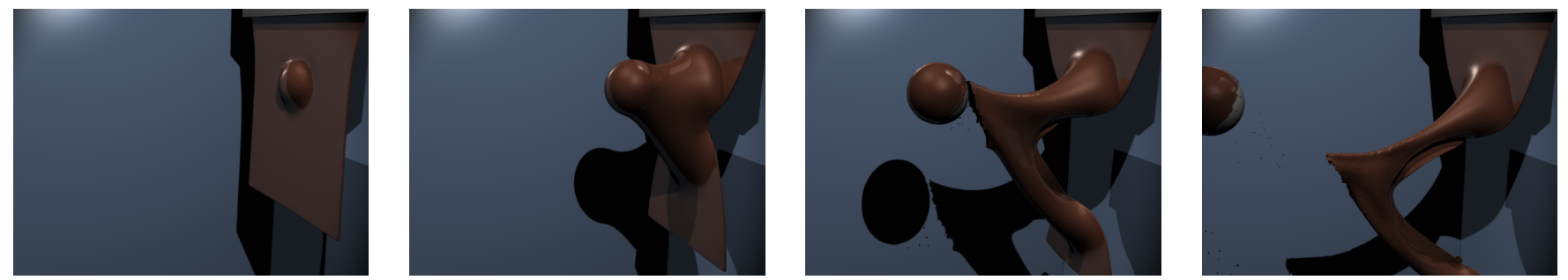

Figure 11: A fast-moving spherical projectile tears through a pouring viscous chocolate sheet.

algorithms. Finally, we hope to extend this approach to fully inviscid sheets, in which the role of remeshing and the attendant numerical viscosity become prime candidates for further study.

In recent years, the fluid animation community has become increasingly interested in the challenging problem of capturing thin fluid sheets. This paper presents a dimensionally-reduced alternative to volumetric approaches, and in doing so it raises many questions and opens a broad avenue for a complementary line of investigation.

\section{Acknowledgments}

This research is supported in part by the Sloan Foundation, the NSF (grants CMMI-11-29917, IIS-11-17257, IIS-10-48948, IIS09-16129, CCF-06-43268), and generous gifts from Adobe, Autodesk, Intel, mental images, NVIDIA, Side Effects Software, and The Walt Disney Company. The first author is supported by a Banting Postdoctoral Fellowship.

\section{References}

ANDo, R., AND Tsuruno, R. 2011. A particle-based method for preserving fluid sheets. In Symposium on Computer Animation, $7-16$.

BARAFF, D., AND WitKIn, A. 1998. Large steps in cloth simulation. In SIGGRAPH, vol. 32, 43-54.

Bargteil, A. W., Hodgins, J. K., Wojtan, C., And Turk, G. 2007. A finite element method for animating large viscoplastic flow. ACM Trans. Graph. (SIGGRAPH) 26, 3, 16.

BAtchelor, G. K. 1967. An Introduction to Fluid Dynamics. Cambridge University Press.

BATTY, C., AND BRIDSON, R. 2008. Accurate viscous free surfaces for buckling, coiling, and rotating liquids. In Symposium on Computer Animation, 219-228.

Batty, C., And Houston, B. 2011. A simple finite volume method for adaptive viscous liquids. In Symposium on Computer Animation, 111-118.

Benjamin, T. B., And Mullin, T. 1988. Buckling instabilities in layers of viscous liquid subjected to shearing. J. Fluid Mech.1 $195,523-540$.
Bergou, M., Wardetzky, M., Robinson, S., Audoly, B., AND GRINSPUN, E. 2008. Discrete elastic rods. ACM Trans. Graph. (SIGGRAPH) 27, 3, 63.

Bergou, M., Audoly, B., Vouga, E., Wardetzky, M., And GrINSPUN, E. 2010. Discrete viscous threads. ACM Trans. Graph. (SIGGRAPH) 29, 4, 116.

Bertails, F., Audoly, B., Cani, M.-P., Leroy, F., QuerLEUX, B., AND LÉvÊQUE, J.-L. 2006. Super-helices for predicting the dynamics of natural hair. ACM Trans. Graph. (SIGGRAPH) 25, 3 (July), 1180-1187.

Bridson, R., Marino, S., And Fedkiw, R. 2003. Simulation of clothing with folds and wrinkles. In Symposium on Computer Animation, Eurographics Association, 28-36.

BRIDSON, R. 2008. Fluid Simulation for Computer Graphics. A. K. Peters, Ltd.

BROCHU, T., AND BRIDSON, R. 2009. Robust topological operations for dynamic explicit surfaces. SIAM J. Sci. Comput. 31, 4, 2472-2493.

Brochu, T., BATty, C., AND BRIDSON, R. 2010. Matching fluid simulation elements to surface geometry and topology. ACM Trans. Graph. (SIGGRAPH) 29, 4, 47.

Buckmaster, J. D., A. Nachman, And Ting, L. 1975. The buckling and stretching of a viscida. J. Fluid Mech. 69, 1, 1-20.

Carlson, M., Mucha, P. J., VAn Horn, R., And Turk, G. 2002. Melting and flowing. In Symposium on Computer Animation, 167-174.

Chentanez, N., Feldman, B. E., Labelle, F., O’Brien, J. F., AND SHEWCHUK, J. R. 2007. Liquid simulation on lattice-based tetrahedral meshes. In Symposium on Computer Animation, 219-228.

DA Silleira, R., Chaieb, S., and Mahadevan, L. 2000. Rippling instability of a collapsing bubble. Science 287, 5457, 1468-1471.

ENGLish, R. E., AND BRIDson, R. 2008. Animating developable surfaces using nonconforming elements. ACM Trans. Graph. (SIGGRAPH) 27, 3, 66. 
Erleben, K., Misztal, M., and Baerentzen, A. 2011. Mathematical foundation of the optimization-based fluid animation method. In Symposium on Computer Animation, 101-110.

Garg, A., Grinspun, E., Wardetzky, M., And Zorin, D. 2007. Cubic shells. In Symposium on Computer Animation, 9198.

Gingold, Y., Secord, A., Han, J. Y., Grinspun, E., And ZORIN, D. 2004. A discrete model for inelastic deformation of thin shells. Tech. rep., New York University.

Grinspun, E., Hirani, A. N., Schröder, P., And Desbrun, M. 2003. Discrete shells. In Symposium on Computer Animation, Eurographics Association, 62-67.

Hasegawa, S., AND FujiI, N. 2003. Real-time rigid body simulation based on volumetric penalty method. In HAPTICS 2003, 326.

Howell, P. D. 1996. Models for thin viscous sheets. European Journal of Applied Mathematics 7, 321-343.

Hutchinson, D., Preston, M., And Hewitt, T. 1996. Adaptive refinement for mass/spring simulations. In Eurographics Workshop on Computer Animation and Simulation, 31-45.

Kass, M., AND Miller, G. 1990. Rapid, stable fluid dynamics for computer graphics. In SIGGRAPH, 49-57.

Kharevych, L., Yang, W., Tong, Y., Kanso, E., Marsden, J. E., Schröder, P., AND Desbrun, M. 2006. Geometric, variational integrators for computer animation. In Symposium on Computer Animation, 43-51.

Martin, S., Kaufmann, P., Botsch, M., Grinspun, E., AND Gross, M. 2010. Unified simulation of elastic rods, shells, and solids. ACM Trans. Graph. (SIGGRAPH) 29, 4, 39.

Misztal, M., Bridson, R., Erleben, K., Baerentzen, A., AND ANTON, F. 2010. Optimization-based fluid simulation on unstructured meshes. In VRIPHYS.

Müller, M., Charypar, D., And Gross, M. 2003. Particlebased fluid simulation for interactive applications. In Symposium on Computer Animation, 154-159.

Müller, M., Keiser, R., Nealen, A., Pauly, M., Gross, M., AND AleXA, M. 2004. Point-based animation of elastic, plastic, and melting objects. In Symposium on Computer Animation, 141-151.

Nealen, A., Müller, M., Keiser, R., Boxerman, E., And CARLSON, M. 2006. Physically based deformable models in computer graphics. Computer Graphics Forum 25, 4, 809-836.

PAI, D. K. 2002. STRANDS: Interactive simulation of thin solids using Cosserat models. Computer Graphics Forum (Eurographics) 21, 3, 347-352.

Pearson, J. R. A., And Petrie, C. J. S. 1970. The flow of a tubular film. Part 1: Formal mathematical representation. $J$. Fluid Mech. 40, 1, 1-19.

RADOVITZKY, R., AND ORTIZ, M. 1999. Error estimation and adaptive meshing in strongly nonlinear dynamic problems. Comput. Methods Appl. Mech. Eng 172, 1-4, 203-240.

Rasmussen, N., Enright, D., Nguyen, D., Marino, S., Sumner, N., Geiger, W., Hoon, S., ANd Fedkiw, R. 2004. Directable photorealistic liquids. In Symposium on Computer Animation, 193-202.
RaYleigh, J. W. S. 1945. Theory of Sound, vol. 2. Dover Publications.

RIBE, N. 2001. Bending and stretching of thin viscous sheets. Journal of Fluid Mechanics 433, 135-160.

RIBE, N. 2002. A general theory for the dynamics of thin viscous sheets. J. Fluid Mech. 457, 255-283.

RIBE, N. 2003. Periodic folding of viscous sheets. Physical Review E 68, 3, 036305.

SAVva, N. 2007. Viscous fluid sheets. PhD thesis, Massachusetts Institute of Technology.

Skorobogatiy, M., And Mahadevan, L. 2000. Folding of viscous sheets and filaments. Europhysics Letters 52, 5, 532538.

SPILlman, J., AND TESChner, M. 2007. CORDE: Cosserat rod elements for the dynamic simulation of one-dimensional elastic objects. In Symposium on Computer Animation, 63-72.

Stokes, G. G. 1845. On the theories of the internal friction of fluids in motion, and of the equilibrium and motion of elastic solids. Transactions of the Cambridge Philosophical Society. Vol. 8 .

Teichman, J., And Mahadevan, L. 2003. The viscous catenary. Journal of Fluid Mechanics 478, 71-80.

Teichmann, J. A. 2002. Wrinkling and sagging viscous sheets. $\mathrm{PhD}$ thesis, MIT.

Villard, J., AND BOROUCHAKI, H. 2005. Adaptive meshing for cloth animation. Engineering with Computers 20, 4, 333-341.

WAng, H., O’Brien, J. F., And Ramamoorthi, R. 2010. Multi-resolution isotropic strain limiting. ACM Trans. Graph. (SIGGRAPH Asia) 29, 6, 156.

Wicke, M., Steinemann, D., And Gross, M. 2005. Efficient animation of point-sampled thin shells. Computer Graphics Forum (Eurographics) 24, 3, 667-676.

Wicke, M., Ritchie, D., Klingner, B. M., Burke, S., Shewchuk, J. R., AND O'BRIEN, J. F. 2010. Dynamic local remeshing for elastoplastic simulation. ACM Trans. Graph. (SIGGRAPH) 29, 4, 49.

Wojtan, C., And TURK, G. 2008. Fast viscoelastic behavior with thin features. ACM Trans. Graph. (SIGGRAPH) 27, 3, 47.

Wojtan, C., Thuerey, N., Gross, M., And Turk, G. 2009. Deforming meshes that split and merge. ACM Trans. Graph. (SIGGRAPH) 28, 3, 76.

Wojtan, C., Thuerey, N., Gross, M., And Turk, G. 2010. Physically-inspired topology changes for thin fluid features. ACM Trans. Graph. (SIGGRAPH) 29, 3.

YAnG, H. T. Y., SAigal, S., Masud, A., And Kapania, R. K. 2000. A survey of recent shell finite elements. Int. J. Numer. Methods Eng., 47, 101-127.

ZHANG, D., AND Yuen, M. M. F. 2001. Cloth simulation using multilevel meshes. Computers and Graphics 25, 3, 383-389.

Zhang, Y., Wang, H., WANG, S., Tong, Y., AND Zhou, K. 2011. A deformable surface model for real-time water drop animation. IEEE TVCG 99. 